\title{
NEGATIVE CURVATURE AND COMBINATORIAL GROUP THEORY
}

\author{
Dedicated to the memory of Hanna Neumann
}

JOSEPH A. WOLF

(Received 29 February 1972)

Communicated by M. F. Newman

Combinatorial group theory has roots in Poincaré's work on the topology of manifolds, which in turn was based on problems in differential equations and analytic number theory. Thus the Fuchsian groups, which are the fundamental (first homotopy) groups of oriented negatively curved conpact surfaces, served as important models in their day. In the last few years there have been advances in the understanding of the structure of fundamental groups of negatively curved manifolds, some of them based on examples from analytic number theory. Here I describe one of these developments and pose a few difficult combinatorial questions.

$M$ is an $n$-dimensional convex simply connected Riemannian manifold with every sectional (Riemannian) curvature $K \leqq 0$. Its rank $r$ is the maximum of the dimensions of the complete flat $(K \equiv 0)$ totally geodesic submanifolds. $\Gamma$ is a group of isometries of $M$ that acts discontinuously and has the property: if $\gamma \in \Gamma$ then the displacement function $d_{\gamma}(x)=$ distance $(x, \gamma x)$ achieves its minimum on $M$. That happens, for example, whenever the quotient space $\Gamma \backslash M$ is compact.

The finite subgroups of $\Gamma$ are just the subgroups that have fixed points on $M$. Thus we have equivalence of (i) $\Gamma$ is torsion free, (ii) $\Gamma \backslash M$ is a manifold and (iii) $\Gamma$ acts freely on $M$. A geodesic ray argument proves $M$ contractible. If $\Gamma$ is torsion free, now $M \rightarrow \Gamma \backslash M$ is a covering by an acyclic space, so the Eilenberg-Maclane cohomology groups $H^{q}(\Gamma)$ are canonically isomorphic to the integral cohomology groups $H^{q}(\Gamma \backslash M ; Z)$; in particular $H^{q}(\Gamma)=0$ for $q>n$. If $\Gamma$ is torsion free and $\Gamma \backslash M$ is compact, then also $\Gamma$ is finitely presented and $H^{q}(\Gamma)$ is the product of a free abelian group $A_{q}$ of $b_{q}<\infty$ generators with a finite abelian group $T_{q}$; if also $\Gamma \backslash M$ is orientable then $H^{n}(\Gamma) \cong Z$ and Poincaré duality says $b_{q}=b_{n-q}$.

The latest information on solvable subgroups of $\Gamma$ is found in [1]. See the Introduction there for a historical account. Let $\Sigma$ be a solvable subgroup of $\Gamma$. Then $\Sigma$ has a finite normal subgroup $\Phi$ such that $\Sigma / \Phi$ is a finite extension of a

Partially supported by an N. S. F. Grant. 
free abelian group $A / \Phi$ on $\leqq r$ generators. Further $A / \Phi$ can be chosen to be a maximal abelian subgroup of $\Sigma / \Phi$. In particular $\Sigma$ is finitely r resented, in fact polycyclic. If $\Gamma \backslash M$ is a manifold, then of course $\Phi=\{1\}$ so $\Sigma$ is a crystallographic group in dimension $\leqq r$.

Problem 1. Let $n>r>0$ integers. Let $G$ be a torsion free countable group in which (a) $H^{q}(G)=0$ for $q>n$ and (b) every solvable sutgroup is a crystallographic group in dimension $\leqq r$. What can one say about $G$ ? For example, if $G$ is not a finite extension of a solvable group, (1) is $G$ a linear grcup? (2) does $G$ contain a nonabelian free group? (3) does every finitely generated subgroup of $G$ have exponential growth ([2], [3])? Does it help to assume (c) that $G$ is finitely presented, that $H^{n}(G) \cong Z$, and that $b_{q}=b_{n-q}<\infty$ for $0 \leqq q \leqq n$ ?

The geometric situation is much simpler when $r=1$, i.e. when $M$ has no totally geodesic submanifold isometric to the Euclidean plane. Of course that includes the case where $M$ has every sectional curvature $K<0$. If $r=1$ and $\Gamma \backslash M$ is a manifold, then every solvable sutgroup of $\Gamma$ is cyclic. At this point we reformulate the problem.

Problem 2. Let $n>1$ integer. Let $G$ te a torsion-free countable group such that $H^{q}(G)=0$ for $q>n$ and such that every solvable subgroup of $G$ is cyclic. What can one say about the structure of $G$ ? What if $G$ is finitely presented, the $H^{q}(G)$ are finitely generated, $H^{n}(G)$ is infinite cyclic and $b_{q}=b_{n-a}$ for $0 \leqq q \leqq n$ ?

Information on these problems would have direct consequences for negatively curved manifolds. On the other hand it seems probable that group-theoretic requirements will force further conditions to be assumed. Those group-theoretic conditions would then be interesting problems in Riemannian geometry. That is the beauty of the subject.

\section{References}

[1] D. Gromoll and J. A. Wolf, 'Some relations between the metric structure and the algebraic structure of the fundamental group in manifolds of nonpositive curvature', Bull. Amer. Math. Soc. 77 (1971), 545-552.

[2] J. Milnor, 'Growth of finitely generated solvable groups', J. Differential Geometry 2 (1968), 447-449.

[3] J. A. Wolf, 'Growth of finitely generated solvable groups and curvature of Riemannian manifolds', J. Differential Geometry 2 (1968), 421-446.

University of California

Berkeley, California 94720

U.S.A. 